BNL.62187

Informal Report

\title{
Department of Energy \\ Review of Laboratory Programs for Women \\ Points-of-Contact Committee
}

Comparative Report

June, 1995

Report written by:

Victoria McLane, Brookhaven National Laboratory

Abbie Layne, Morgentown Energy Technology Center

Co-Chairs, DOE Review of Laboratory Programs for Women Point-of-Contacts

Data Compiled by:

Debra Duke, Kim Magrini

National Renewable Energy Laboratory

Victoria McLane

Brookhaven National Laboratory

Karen Wieda

Pacific Northwest Laboratories

DiSTRIBUTION OF THIS DOCUMENT IS UNLIMITED/PP// 


\section{Table of Contents}

Introduction

Statistical Data

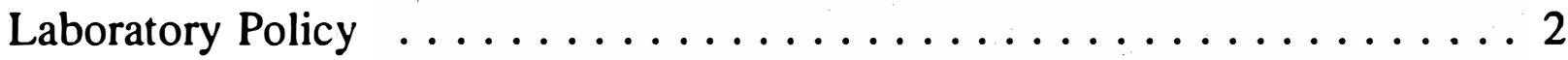

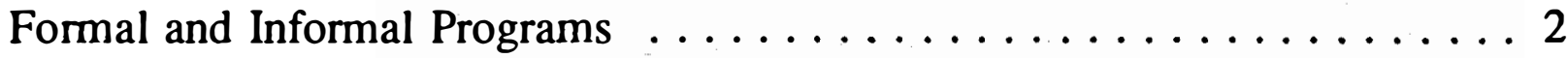

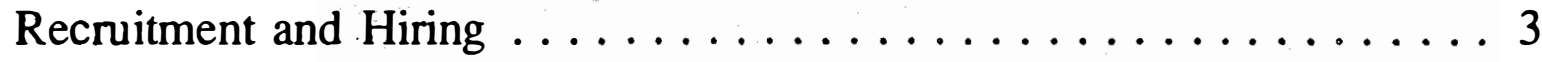

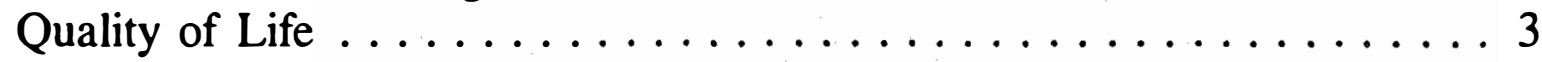

Career Development and Advancement ............... 3

Women's Programs/Women's Program Coordinator ...........4

Educational Programs $\ldots \ldots \ldots \ldots \ldots \ldots \ldots \ldots \ldots$

Appendix A: Statistical Data

Appendix B: Laboratory Policy

Appendix C: Formal and Informal Programs

Appendix D: Educational Programs

Appendix E: List of Participating Facilities

\section{DISCLAIMER}

This report was prepared as an account of work sponsored by an agency of the United States Government. Neither the United States Government nor any agency thereof, nor any of their employees, makes any warranty, express or implied, or assumes any legal liability or responsibility for the accuracy, completeness, or usefulness of any information, apparatus, product, or process disclosed, or represents that its use would not infringe privately owned rights. Reference herein to any specific commercial product, process, or service by trade name, trademark, manufacturer, or otherwise does not necessarily constitute or imply its endorsement, recommendation, or favoring by the United States Government or any agency thereof. The views and opinions of authors expressed herein do not necessarily state or reflect those of the United States Government or any agency thereof. 


\section{Introduction}

The mission of the DOE Review of Laboratory Programs for Women is to:

1) provide DOE and its Laboratories with effective strategies, targeting women, for establishing aggressive outreach programs which improve the access of women to careers in science, engineering, and mathematics.

2) ensure that the Department and its Laboratories are exemplary places of employment by providing programs which enhance opportunity, remove barriers, and assist women in achieving full professional development.

A survey of the DOE facilities was undertaken by the Points-of-Contact for the DOE Review of Laboratory Programs for Women in order to gather data to be used as a baseline against which to measure future progress. We plan to look at current programs already in place and evaluate them with a view to deciding which programs are most effective, and selecting model programs suitable for implementation at other facilities.

The survey focused on four areas: 1) statistical data, 2) laboratory policy, 3) formal and informal programs which affect the quality of life in the work environment, and career development and advancement, and 4) educational programs.

Although this report focuses on women, the problems discussed affect all DOE facility employees.

\section{Statistical Data}

To quantify the problem of recruiting women into the scientific and technological fields, and to measure their progress, data must be gathered on the distribution of women at the DOE facilities relative to the population as a whole. Criteria must be developed to make the comparison of the data as valid as possible.

A Statistical Database Survey was prepared and sent to all DOE facilities. The goal of the Survey was to establish baseline data against which meaningful comparisons about the status of gender equity among the facilities can be made. Comparisons of women versus total staff were requested by job categories, educational levels, ethnicity, and pay-bands. Twenty facilities comprising almost 43,000 employees responded. Of those responding, twelve submitted incomplete data. 
Based on this data we find that for all 20 facilities:

- Total female representation is $30 \%$.

- Female representation on research staff is $15 \%$.

- Female representation on administrative staff is $52 \%$.

- Most females occupy the 3 lowest pay-bands.

Complete information is found in the report by D. Duke and K. Magrini, "DOE Comparative Salary Survey Report", April 18, 1995. Selected tables from this report are given in Appendix A.

We recommend that future surveys include statistics by discipline along with the available population by discipline, years since last degree, and years in workforce. Information on RIF's should also be included.

\section{Laboratory Policy}

Twenty-four facilities were surveyed regarding policies affecting women. The results are tabulated in Appendix B.

Improvements to this survey to be undertaken in the coming year should include:

- An attempt to define "top" management, so that the statistics are comparable across the facilities surveyed.

- Data on the percentage of women on various committees at the facilities, e.g., search committees.

- Data on the percentage of women speakers on scientific topics at the facilities.

\section{Formal and Informal Programs}

In order to obtain the participation of women and minorities at all levels, including the highest levels, the DOE facilities must create a non-hostile environment, and institute programs which assist women in their professional development.

We have examined programs in the following areas:

1) Recruitment and Hiring

2) "Quality of Life"

3) Career Development and Advancement

4) Women's Programs

All twenty-four facilities surveyed responded. The results are tabulated in Appendix C. 


\section{Recruitment and Hiring}

All facilities responding have an active policy to recruit and hire women. However, more detailed information must be gathered to identify what strategies are working and what are not.

Professional intern programs and re-entry/retraining programs are of special interest to women who often take time out from their careers to care for their young children. Only a few facilities have such programs in place.

\section{Quality of Life}

Women have traditionally been and still are the primary care-givers in the family. The migration of women into the work force has increased the pressure on both men and women with young children, aging parents, or other care responsibilities. It is especially important for two-career families to have reliable child care and elder care, as well as family leave policies which allow maternity and adoption leave, and leave for care of sick or disabled family members. Only eight of the responding facilities provide child-care facilities; facilities are planned for three other facilities. All of the facilities have some form of Maternity/Patemity or Family Leave, and most of these include adoption. Three facilities also include adoption subsidies in their benefits packages.

Alternate work schedules, such as flex-time, compressed work weeks, tele-commuting, and jobsharing need to be implemented to ease the burden of competing priorities. Seventeen of the facilities have a provision for flexible work schedules; twelve provide a compressed work week and/or tele-commuting.

Hostility in the work place affects the morale, and therefore effectiveness, of all employees. Programs must be in place to help sensitize all employees to gender and cultural differences, and their value in the work place. Sexual harassment workshops should be given to all employees. Sixteen of the reporting facilities have some form of Gender/Cultural Differences Workshop, however, only a few target all employees. Twenty-two facilities have Sexual Harassment Workshops; of these, sixteen target all employees.

Employee assistance programs are in place at all the facilities, and most have some form of wellness program.

\section{Career Development and Advancement}

Of equal importance with recruiting and hiring in obtaining workforce diversity is the advancement of women already on the staff. Of major importance in achieving this goal is encouraging personal development and improving the "quality of life" in the workplace.

Informal mentoring has always existed. However, because most people in supervisory and management positions are white males, and because most people feel most comfortable with 
those who are "like themselves", women and minorities have been largely left out of the informal mentoring process. Formal mentoring programs increase the access of women to information useful to doing their job effectively, and to advancing their careers. Ten of the facilities responding currently have career development mentoring programs; another six are looking into implementing a program. Only one of the facilities has new employee mentoring program, and two others are looking into implementing one.

About half of the facilities have career planning programs, a program we believe should be in place at every facility.

Most facilities have tuition reimbursement programs, with most of these paying $100 \%$ for jobrelated studies.

\section{Women's Programs/Women's Program Coordinator}

A women's advocate acts as a focus for women and a voice for their concerns. The previous reviews have all recommended that all to the DOE contractor facilities identify or appoint a Women’s Program Coordinator. Only seven of the respondents have such a position.

Interacting with one's peers is important and support groups should be encouraged until a "critical mass" of women is reached at all levels of the facility structure. Half of the facilities reporting have a women's group.

\section{Educational Outreach}

To increase the number of women entering scientific and technological fields, strong programs, targeting female students, are needed to attract a talented pool of students and to encourage them to continue their studies in math and science. The DOE must develop a system for tracking students who attend educational outreach programs at DOE facilities from primary school through graduate school in order to determine what works.

A survey of the programs currently in place at the DOE facilities who responded to the survey is given in Appendix D. Additional information on the percentage of women participating in the various programs will be gathered over the coming year. 


\section{Appendix A}

\section{Statistical Data}




\section{Detailed Workforce Breakdown}

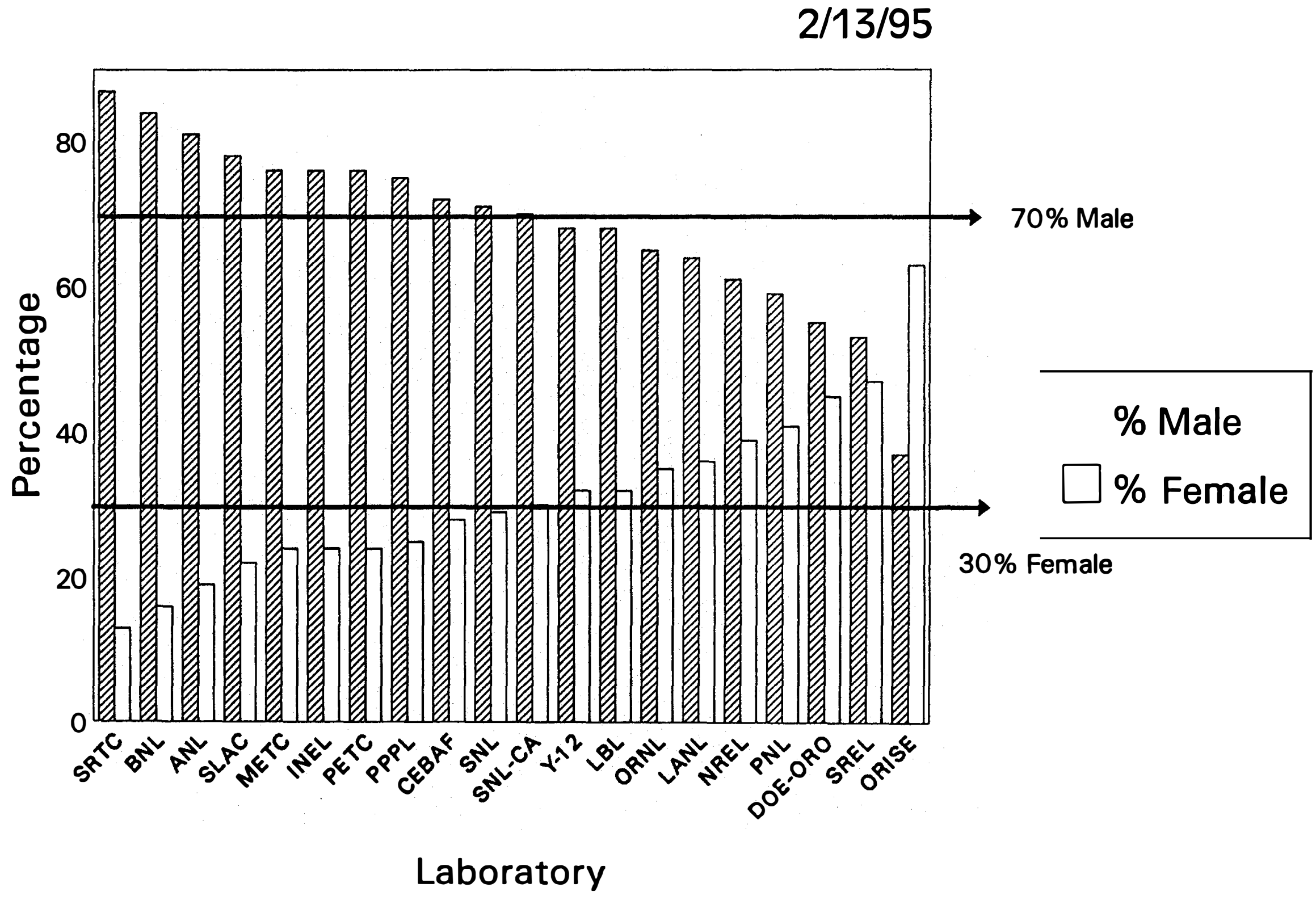




\section{DOE Review of Laboratory Programs for Women Workforce Breakdown for Administration}

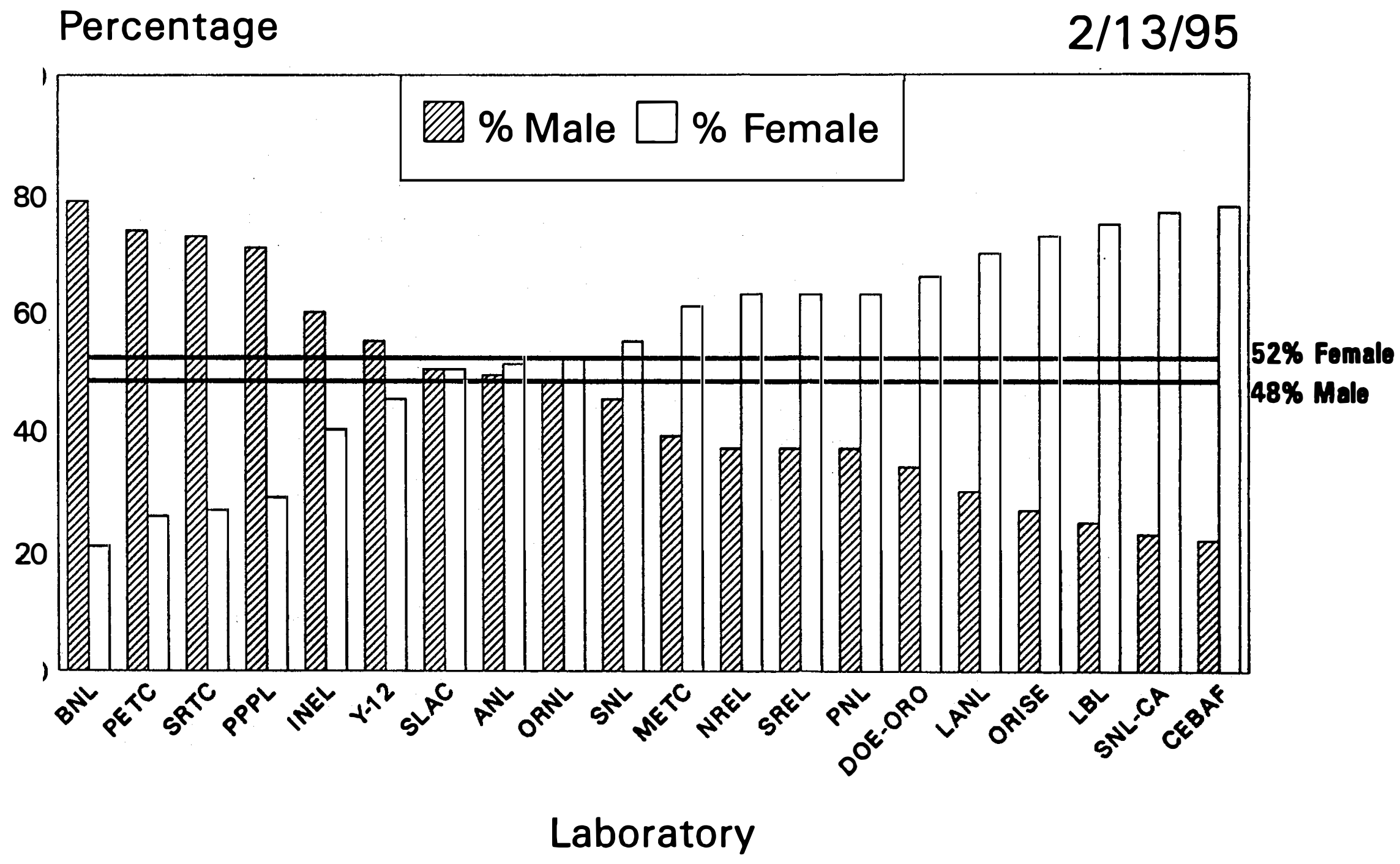


DOE Review of Laboratory Programs for Women Workforce Breakdown for Research

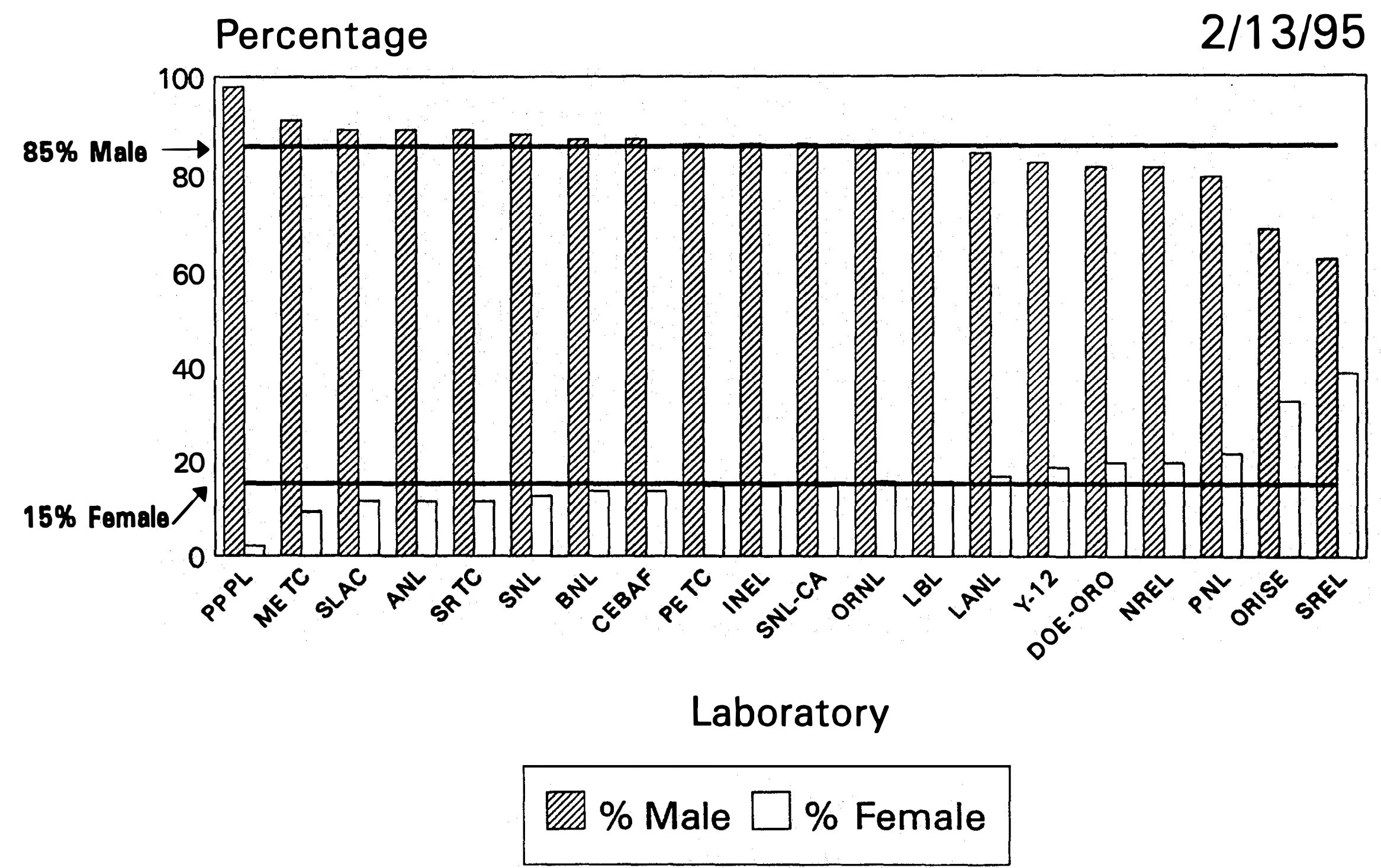




\section{Workforce Breakdown by Pay Ranges/Research}

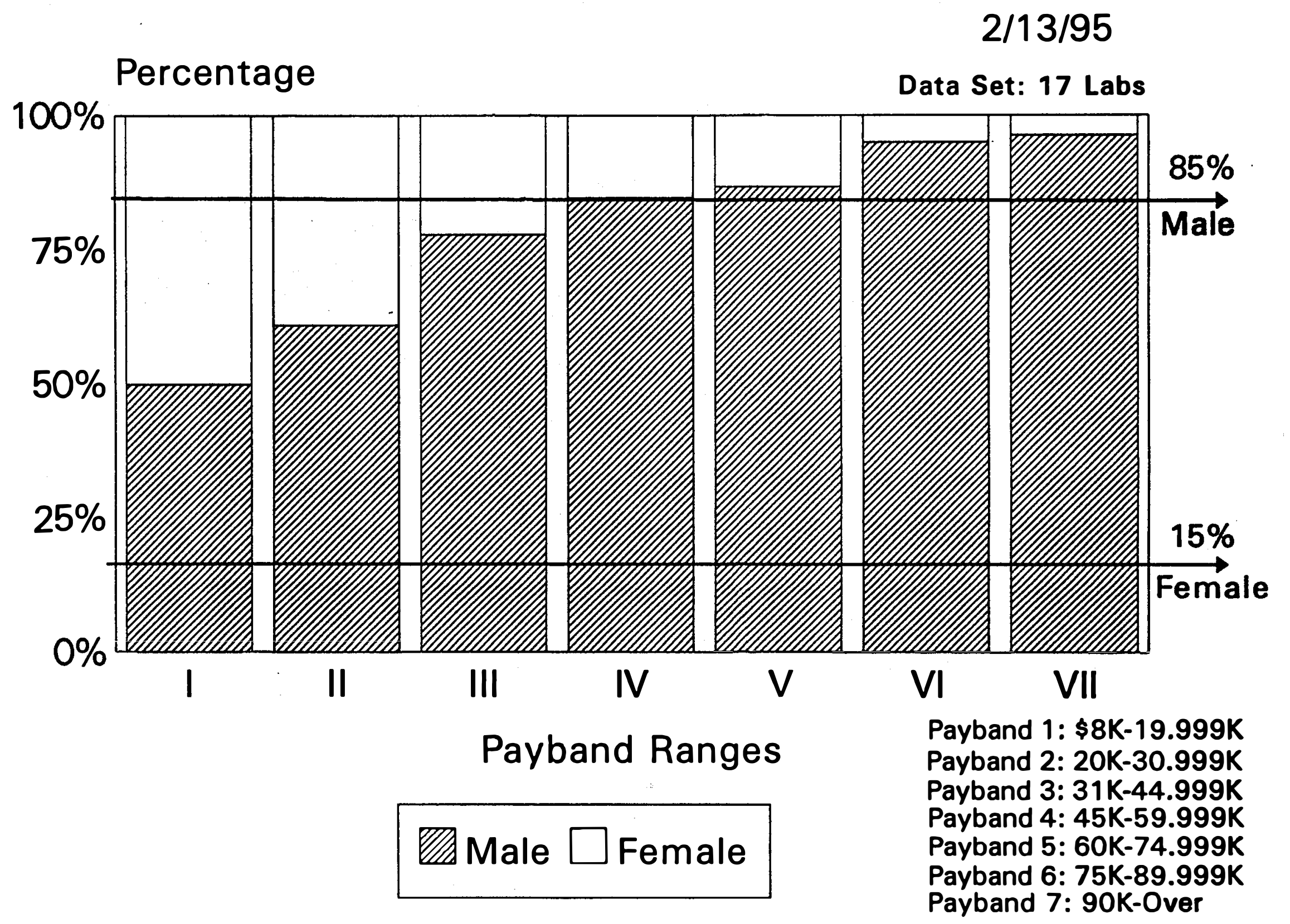

National Renewable Energy Laboratory Duke/Magrini 


\section{Workforce Breakdown by Pay Ranges/Administration}

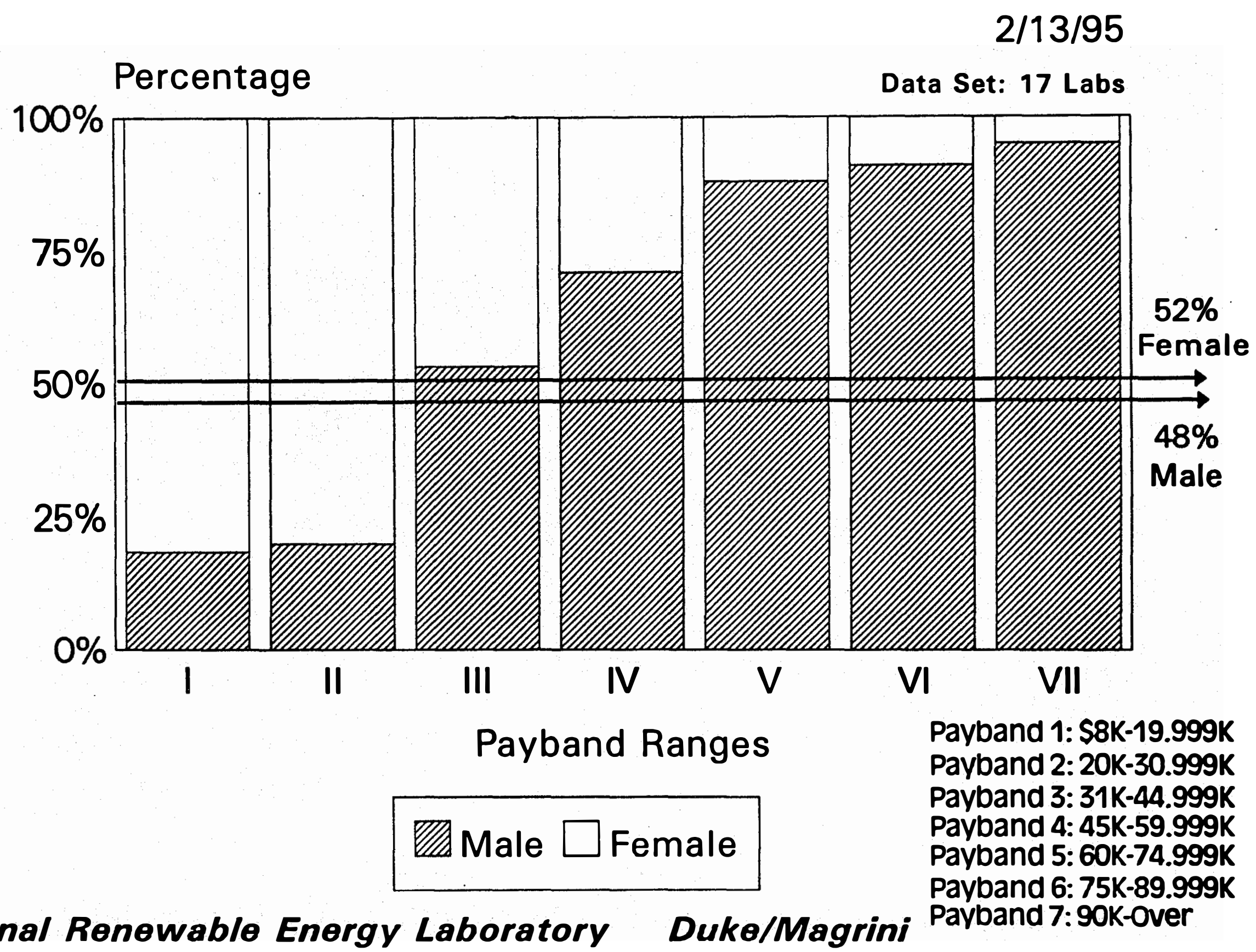




\section{Workforce Degree Breakdown}

Thousands

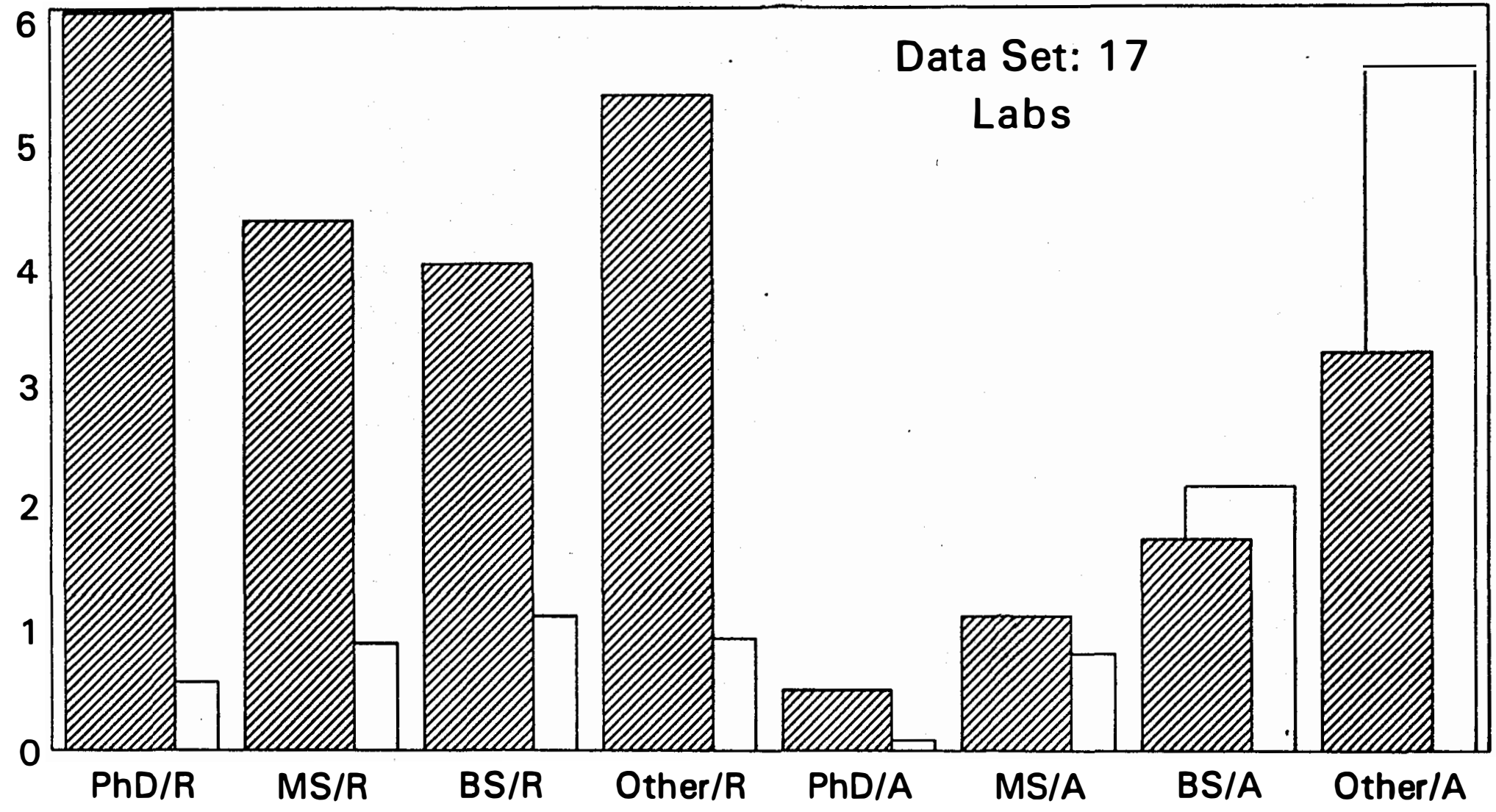

$\mathrm{R}=$ Research

Degree Type

$A=$ Administration

\section{Male $\square$ Female}

$M S=M S+M A$

$\mathrm{BS}=\mathrm{BS}+\mathrm{BA}$

Other $=$ AS + Other 


\section{Workforce EEO Breakdown}

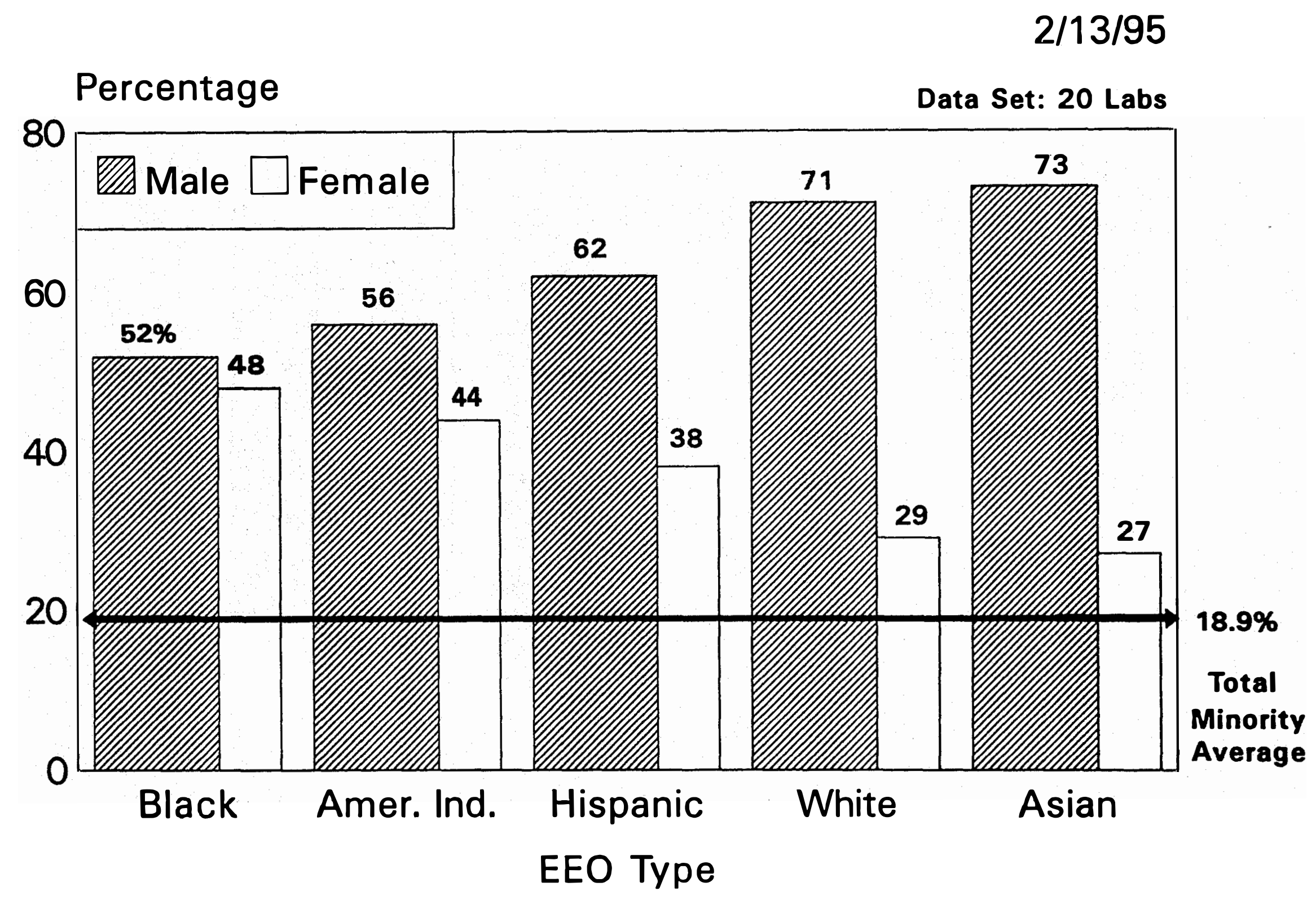




\section{Appendix B}

\section{Laboratory Policy}




\begin{tabular}{|c|c|c|c|c|c|c|c|c|c|c|c|c|c|c|c|c|c|c|c|c|c|c|c|c|}
\hline $\begin{array}{l}\text { Laboratory } \\
\text { Pollcy }\end{array}$ & 题 & $\frac{1}{\alpha}$ & $\underset{\mathbf{D}}{\overrightarrow{\mathbf{z}}}$ & 訔 & $\sum_{w}^{0}$ & $\frac{1}{x}$ & $\underline{\underline{w}}$ & そ & $\overrightarrow{\underline{a}}$ & $\underset{J}{\vec{z}}$ & $\frac{0}{2}$ & $\frac{N}{\frac{N}{\Sigma}}$ & 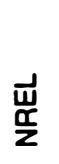 & 崖 & 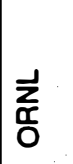 & $\frac{0}{\underline{w}}$ & $\frac{1}{2}$ & $\frac{a}{a}$ & $\varliminf_{\text {क }}^{0}$ & ঠ্ & 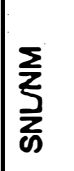 & 岕 & $\mid \begin{array}{l}0 \\
\frac{6}{6} \\
\text { c. }\end{array}$ & $\frac{0}{1}$ \\
\hline $\begin{array}{l}\text { Active policy to recruit women } \\
\text { Person responsible. } \\
\text { Plan in operation. } \\
\text { Measures of success. }\end{array}$ & 8 & 1 & $\begin{array}{l}1 \\
1\end{array}$ & $\begin{array}{l}1 \\
1\end{array}$ & 1 & 1 & $\begin{array}{l}1 \\
1 \\
1\end{array}$ & 1 & 1 & 1 & $\begin{array}{l}1 \\
1 \\
1\end{array}$ & 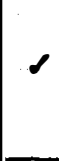 & 1 & $\begin{array}{l}1 \\
1 \\
1\end{array}$ & $\begin{array}{l}1 \\
1 \\
1\end{array}$ & $\begin{array}{l}1 \\
1 \\
1\end{array}$ & 2 & & 8 & $\begin{array}{l}1 \\
1 \\
1\end{array}$ & $\begin{array}{l}1 \\
1 \\
1\end{array}$ & 1 & $\begin{array}{l}1 \\
1 \\
1\end{array}$ & 1 \\
\hline $\begin{array}{l}\text { Active policy to hire women } \\
\text { Person responsible. } \\
\text { Plan in operation. } \\
\text { Measures of success. } \\
\end{array}$ & 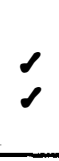 & 1 & $\begin{array}{l}1 \\
1 \\
1\end{array}$ & & $\begin{array}{l}1 \\
1 \\
\end{array}$ & $\begin{array}{l}1 \\
1 \\
2\end{array}$ & 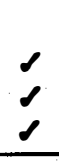 & $\begin{array}{l}1 \\
\end{array}$ & $\begin{array}{l}1 \\
1 \\
1\end{array}$ & 2 & $\begin{array}{l}1 \\
1 \\
1\end{array}$ & 1 & 1 & $\begin{array}{l}1 \\
1 \\
1\end{array}$ & $\begin{array}{l}1 \\
1 \\
1\end{array}$ & $\begin{array}{l}1 \\
1 \\
1\end{array}$ & $\begin{array}{l}1 \\
1 \\
1\end{array}$ & & 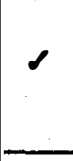 & $\begin{array}{l}1 \\
1 \\
1 \\
\end{array}$ & $\begin{array}{l}1 \\
1 \\
1\end{array}$ & 1 & $\begin{array}{l}1 \\
1 \\
1\end{array}$ & 2 \\
\hline $\begin{array}{l}\text { Active policy to retain women } \\
\text { Person responsible. } \\
\text { Plan in operation. } \\
\text { Measures of success. }\end{array}$ & 8 & 1 & $\begin{array}{l}1 \\
1\end{array}$ & & 1 & 1 & $\begin{array}{l}1 \\
1\end{array}$ & 1 & $\begin{array}{l}1 \\
1 \\
1\end{array}$ & & & & 8 & $\begin{array}{l}1 \\
1 \\
1\end{array}$ & $\begin{array}{l}1 \\
1 \\
1\end{array}$ & $\begin{array}{l}1 \\
1 \\
1\end{array}$ & $\begin{array}{l}1 \\
1 \\
1\end{array}$ & & & 1 & 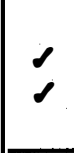 & 1 & $\begin{array}{l}1 \\
1 \\
1\end{array}$ & 1 \\
\hline $\begin{array}{l}\text { Recruitment, hiring, retaining, promoting } \\
\text { women part of management } \\
\text { pertormance appraisal }\end{array}$ & & 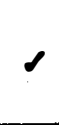 & l & 1 & & & 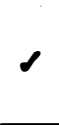 & 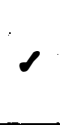 & 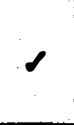 & 8 & o & 1 & & & 8 & $d$ & $d$ & & & 1 & 1 & & 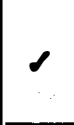 & \\
\hline $\begin{array}{l}\text { Written sexual harassment policy } \\
\text { Person responsible. } \\
\text { Plan in operation. } \\
\text { Measures of success. }\end{array}$ & 8 & $\begin{array}{l}1 \\
1 \\
1\end{array}$ & 8 & 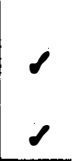 & 1 & 2 & 2 & $\begin{array}{l}1 \\
8 \\
1\end{array}$ & $\begin{array}{l}1 \\
1 \\
1 \\
1\end{array}$ & 1 & 2 & 8 & $\begin{array}{l}1 \\
1 \\
1\end{array}$ & $\begin{array}{l}2 \\
1 \\
1\end{array}$ & $\begin{array}{l}1 \\
1 \\
1\end{array}$ & $d$ & $\begin{array}{l}2 \\
1 \\
1\end{array}$ & 1 & $\begin{array}{l}1 \\
1 \\
1 \\
1\end{array}$ & $\begin{array}{l}1 \\
1 \\
1 \\
1\end{array}$ & $\begin{array}{l}1 \\
1 \\
1\end{array}$ & 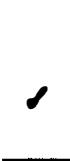 & $\begin{array}{l}1 \\
1 \\
1\end{array}$ & 1 \\
\hline \begin{tabular}{|l} 
Policy which guarentees equal pay for \\
equal work. \\
Person responsible. \\
Plan in operation. \\
Measures of sucess.
\end{tabular} & $s$ & 1 & 2 & 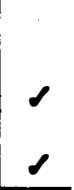 & 1 & 2 & $\begin{array}{l}1 \\
1 \\
1\end{array}$ & 1 & 1 & 12 & & 8 & $\begin{array}{l}1 \\
1 \\
1 \\
1\end{array}$ & $\begin{array}{l}1 \\
1 \\
1\end{array}$ & $\begin{array}{l}1 \\
1 \\
1\end{array}$ & & $\begin{array}{l}1 \\
1 \\
1\end{array}$ & $\begin{array}{l}1 \\
1 \\
1\end{array}$ & $\begin{array}{l}1 \\
1 \\
1\end{array}$ & $\begin{array}{l}1 \\
1 \\
1 \\
1\end{array}$ & $\begin{array}{l}1 \\
1 \\
1\end{array}$ & & $\begin{array}{l}1 \\
1 \\
1 \\
1\end{array}$ & 8 \\
\hline $\begin{array}{l}\text { Women represented in top } \\
\text { management }(\%) \text {. }\end{array}$ & 10 & 4 & 10 & 20 & 7 & 16 & 9.8 & 14 & 12 & 7 & 1 & 4 & & 10 & 2 & 10 & 4 & 0 & .01 & 25 & 12 & 14 & 10 & \\
\hline $\begin{array}{l}\text { Women's committee or spokesperson } \\
\text { consulted regarding major management } \\
\text { decisions. }\end{array}$ & 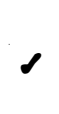 & 1 & $\rho$ & & $J$ & & 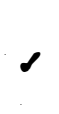 & & $J$ & & 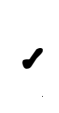 & 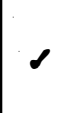 & & & & $d$ & & 8 & & 1 & 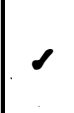 & $d$ & & 1 \\
\hline
\end{tabular}




\section{Appendix C}

\section{Formal and Informal Programs}


Formal and Informal

Programs

Sectlon A.

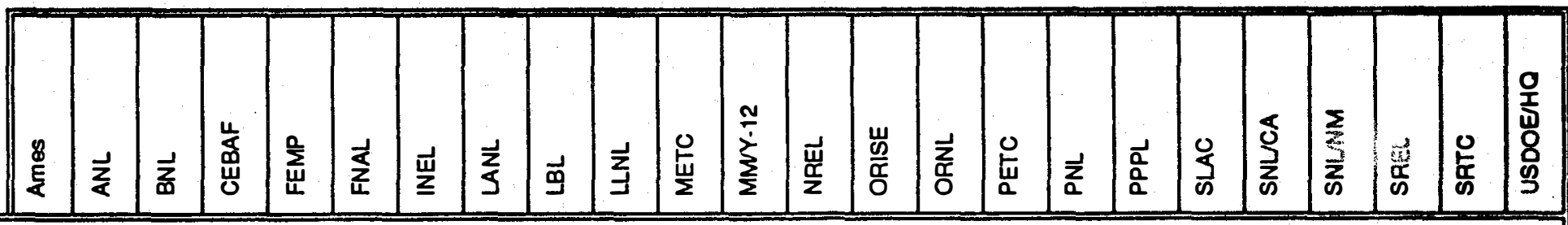

\begin{tabular}{|c|c|c|c|c|c|c|c|c|c|c|c|c|c|c|c|c|c|c|c|c|c|c|c|c|}
\hline \multicolumn{25}{|l|}{ Recrultmont, Hiring Programs } \\
\hline Management Intem Development Program & & & Pn & & & & 1 & 1 & 1 & Pn & & 1 & & & 1 & 1 & & & & & & & Pn & 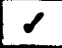 \\
\hline Professional Intem Program & & 1 & $l$ & & & & & & & & 1 & & & & 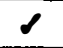 & 1 & & & & & & & & \\
\hline \multicolumn{25}{|c|}{ Altornative Work Schodules (statod policy allowing such) } \\
\hline Flexible Work Schedules & 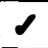 & 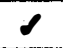 & $\mathbf{S}$ & 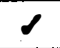 & & $\mathbf{s}$ & & 1 & 1 & 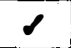 & 1 & 1 & $\mathbf{P}$ & 1 & $\mathbf{P}$ & $\checkmark$ & 1 & 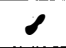 & 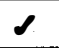 & 1 & 1 & 1 & & 1 \\
\hline Part Time/Job Sharing & 1 & 1 & 1 & & 1 & & 1 & 1 & 1 & 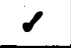 & & 1 & 1 & 1 & 1 & 1 & 1 & & & 1 & 1 & 1 & 1 & 1 \\
\hline Compressed Work Week & & $\mathbf{S}$ & $\mathbf{P}$ & & & & & $\mathbf{S}$ & $\mathbf{P}$ & 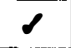 & $\checkmark$ & 1 & 1 & 1 & 1 & 1 & 1 & & & 1 & 1 & & & 1 \\
\hline Telecommuting & & $\mathbf{S}$ & & & & & & & 1 & $\mathbf{P}$ & & Pn & & & Pn & 1 & $\mathbf{s}$ & & 1 & Pn & Pn & & & \\
\hline \multicolumn{25}{|l|}{ Dependent Caro } \\
\hline $\begin{array}{l}\text { Matemity/Patemity/Family Leave (months) } \\
\text { Adoption included (A) }\end{array}$ & & $\begin{array}{c}12 \\
A\end{array}$ & A & $\begin{array}{l}3 \\
\text { A }\end{array}$ & 3 & A & $\begin{array}{l}\mathbf{3} \\
\mathbf{A}\end{array}$ & $\begin{array}{l}4 \\
A\end{array}$ & B & $\begin{array}{l}6 \\
\mathbf{A}\end{array}$ & $?$ & $\begin{array}{l}3 \\
\mathbf{A}\end{array}$ & $\begin{array}{l}6 \\
\text { A }\end{array}$ & $\begin{array}{l}4 \\
A\end{array}$ & A & $?$ & $\begin{array}{l}4 \\
A\end{array}$ & $\begin{array}{c}12 \\
A\end{array}$ & $\begin{array}{l}3 \\
\mathbf{A}\end{array}$ & $\begin{array}{l}12 \\
A\end{array}$ & $\begin{array}{l}12 \\
A\end{array}$ & A & 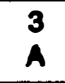 & $?$ \\
\hline $\begin{array}{c}\text { Dependent Home Care Authorized } \\
\text { Absence with Pay (days) }\end{array}$ & 5 & $\stackrel{?}{P L}$ & $\begin{array}{c}5 \\
\text { SL }\end{array}$ & $\begin{array}{c}5 \\
\text { SL }\end{array}$ & 10 & $\begin{array}{l}? \\
F L\end{array}$ & & 30 & $\begin{array}{l}30 \\
\text { SL }\end{array}$ & $\begin{array}{l}30 \\
\text { SL }\end{array}$ & & $\stackrel{?}{\mathrm{FL}}$ & & & $\stackrel{?}{\text { FL }}$ & & $?$ & & $\stackrel{?}{S}$ & Pns & Pn & & 1 & \\
\hline $\begin{array}{l}\text { Child Care Center, Facility Owned } \\
\text { On-site/Off-site (ON/OFF) } \\
\text { Facility/Contractor operated (F/C) }\end{array}$ & & $\begin{array}{c}\text { on } \\
\text { on } \\
c\end{array}$ & $\begin{array}{c}1 \\
\text { on } \\
c\end{array}$ & & & $\begin{array}{c}1 \\
\text { on } \\
\text { C }\end{array}$ & & $\mathbf{s}$ & Pn & $\begin{array}{l}\rho^{\circ} \\
\text { off } \\
\mathrm{C}\end{array}$ & $\begin{array}{c}\text { r } \\
\text { on } \\
c\end{array}$ & & & & & & $\begin{array}{l}\text { Pn } \\
\text { on } \\
\text { C }\end{array}$ & $\begin{array}{c}\text { off } \\
\text { of }\end{array}$ & & off & Pn & & & on \\
\hline Child Care Referral Service & 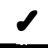 & & Pn & $\mathbf{s}$ & 1 & & & 1 & 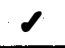 & & & 1 & $\mathcal{L}$ & $j$ & 1 & & 1 & & 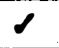 & 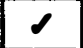 & 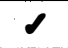 & & & \\
\hline Sick Child Care Facility & $\jmath$ & & & & $?$ & & $?$ & & $\mathbf{R}$ & & & $\mathbf{R}$ & & $\mathbf{R}$ & & & $\mathbf{R}$ & & & & $\mathbf{s}$ & & & \\
\hline Summer Recreation Program & & 1 & 1 & & & 1 & & & 1 & & 1 & 1 & & & & & $\mathbf{R}$ & 1 & & $\cdot$ & 1 & & & \\
\hline Elder Care Facility & & & & & & & & & $\mathbf{R}$ & & & $\mathbf{R}$ & & & & & $R$ & & & $\mathbf{R}$ & $\mathbf{R}$ & & & \\
\hline \multicolumn{25}{|l|}{ Employoe Aselatanco } \\
\hline Employee Assislance Program & 1 & 1 & 8 & 1 & 1 & 1 & 1 & 1 & 1 & 1 & 1 & 1 & 1 & 1 & 1 & 1 & 1 & 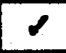 & 1 & 1 & 2 & & 7 & 1 \\
\hline Wellness Program & & 1 & 1 & 1 & 1 & 1 & 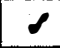 & 1 & 1 & 1 & 1 & 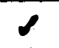 & 1 & 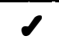 & & 1 & 1 & 1 & 1 & 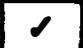 & 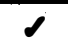 & & $\gamma$ & 2 \\
\hline \multicolumn{25}{|l|}{ Other Bonofits } \\
\hline $\begin{array}{c}\text { Co-insurance for married couples both } \\
\text { employed at facility. }\end{array}$ & $\mathcal{L}$ & 1 & 1 & & & & 1 & 1 & 1 & & & & & & & & & 1 & & & & & $\mathcal{\prime}$ & 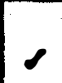 \\
\hline Cafeteria/Flex benefits & & 1 & $H$ & & & 1 & 1 & 1 & 1 & & & $\mathrm{H}$ & 1 & 1 & $\mathrm{H}$ & & 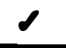 & & 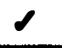 & & & & $\rho$ & 2 \\
\hline Adoption subsidies & & & 1 & & & & & & & & & & & & & & & & & 1 & 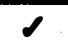 & & & \\
\hline Employee awards & & $\checkmark$ & $\checkmark$ & & & & & 1 & & Pn & & 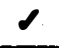 & 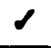 & & 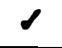 & & 1 & & & 1 & 1 & & & \\
\hline
\end{tabular}


Formal and Informal

Programs

Section B.

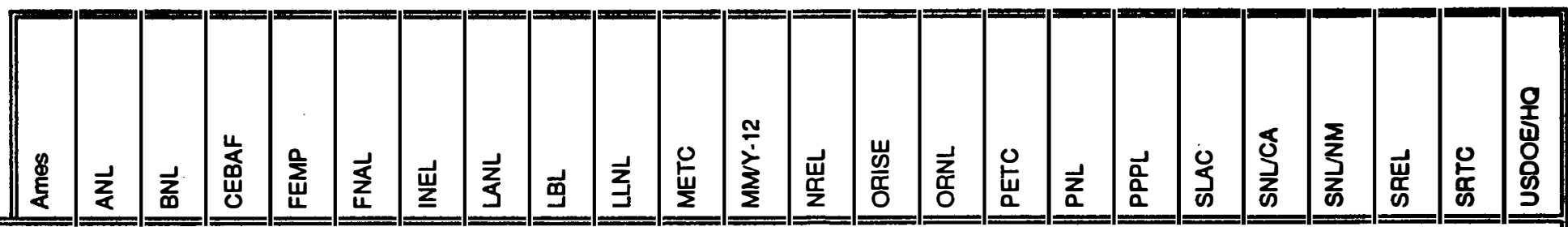

\section{Employeo Education}

Tuition reimbursement Undergraduate \% Job related only (JR)

Tuition reimbursement Graduate \% Job related only (JR)

Workstudy Program

Reentry/Retraining Program

Cereer DovelopmenvCaroer Advancement

\begin{tabular}{|c|c|c|c|c|c|c|c|c|c|c|c|c|c|c|c|c|c|}
\hline Career development mentoring & $\mathbf{S}$ & $\mathbf{s}$ & 1 & $\mathbf{s}$ & $j$ & 1 & 1 & 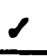 & \lrcorner & & 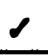 & 1 & 1 & $\mathbf{s}$ & $\mathbf{P}$ & $\mathbf{P}$ & 1 \\
\hline New employee mentoring & $\mathbf{s}$ & & & & & & 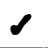 & & & & & 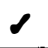 & & $\mathbf{s}$ & & & \\
\hline Career planning & 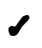 & & 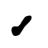 & $\mathbf{s}$ & 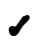 & 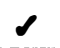 & $\curvearrowright$ & 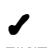 & $\curvearrowright$ & 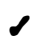 & & 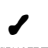 & 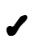 & & $\curvearrowright$ & 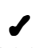 & $\mathcal{L}$ \\
\hline Succession planning & 1 & & & & & PN & $\mathbf{S}$ & 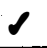 & 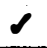 & 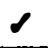 & & $d$ & & Pn & 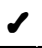 & 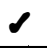 & $\mathcal{1}$ \\
\hline
\end{tabular}

\section{Training Programs for Employees}

\begin{tabular}{|c|c|c|c|c|c|c|c|c|c|c|c|c|c|c|c|c|c|c|c|c|c|c|}
\hline Professional Skills Training & & 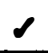 & 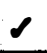 & 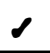 & $\checkmark$ & $\mathbf{s}$ & 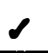 & 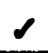 & 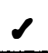 & $\alpha$ & 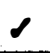 & 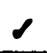 & 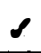 & 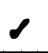 & 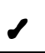 & 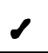 & 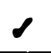 & & 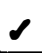 & 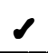 & 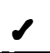 & 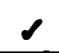 \\
\hline $\begin{array}{l}\text { Management Training } \\
\text { Targets women (T) }\end{array}$ & 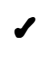 & $\checkmark$ & $\checkmark$ & 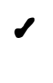 & & & $\checkmark$ & $\checkmark$ & $\checkmark$ & 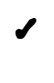 & $\begin{array}{l}1 \\
\end{array}$ & $\checkmark$ & & 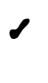 & 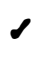 & $\frac{1}{T}$ & $\frac{1}{T}$ & 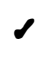 & $\checkmark$ & 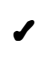 & $\checkmark$ & $\frac{1}{T}$ \\
\hline $\begin{array}{r}\text { Apprenticeship Program } \\
\text { Targets women (T) }\end{array}$ & & $\checkmark$ & & & & & 2 & $\checkmark$ & & $\frac{1}{1}$ & & $\checkmark$ & & & & & & & $s$ & 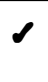 & & \\
\hline
\end{tabular}

\section{Workforos Diveralty}

\begin{tabular}{|c|c|c|c|c|c|c|c|c|c|c|c|c|c|c|c|c|c|c|c|c|}
\hline Workforce Diversity Task Force & 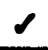 & 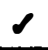 & $\checkmark$ & $\mathbf{P}$ & 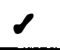 & $\mathbf{P}$ & 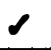 & 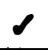 & 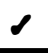 & & 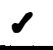 & & 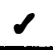 & 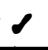 & $s$ & \lrcorner & 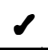 & 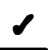 & 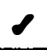 & 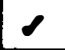 \\
\hline Gender Cultural Differences Workshop & \lrcorner & 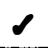 & & Pn & Pn & $\mathbf{P}$ & 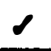 & 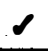 & 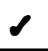 & 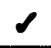 & $s$ & 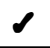 & $\checkmark$ & $\checkmark$ & 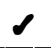 & 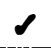 & $\rho$ & $\mathcal{1}$ & $\mathcal{\prime}$ & $\rho$ \\
\hline
\end{tabular}

\section{Soxual Haresement Awareneas Program}

Training for Managers

Training for all employees

Employee Guide

\begin{tabular}{|c|c|c|c|c|c|}
\hline & 1 & 1 & 1 & 1 \\
\hline & 1 & 1 & 1 & 1 & 1 \\
\hline
\end{tabular}


Formal and Informal

Programs

Sectlon C.

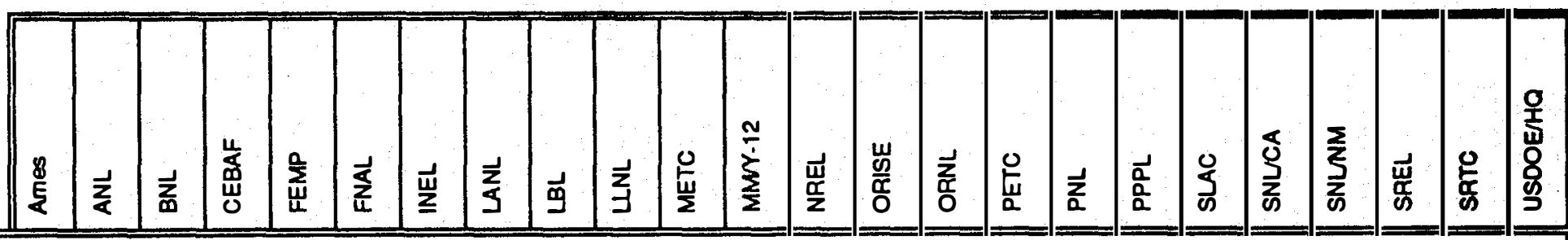

\begin{tabular}{|c|c|c|c|c|c|c|c|c|c|c|c|c|c|c|c|c|c|c|c|c|c|c|c|c|}
\hline \multicolumn{25}{|l|}{ Womon's Programs } \\
\hline $\begin{array}{l}\text { Women's Program Coordinator/Initiator } \\
\text { (FTE) }\end{array}$ & & 0.3 & 0.2 & & & & 1.0 & & & & 1.0 & & & & & 0.3 & & & & & 0.1 & & & 1. \\
\hline Women in Science/Technology Group & WC & $\rho$ & 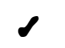 & 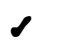 & & & 1 & & 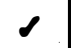 & 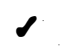 & & 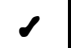 & & & 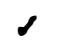 & & $\mathcal{L}$ & & 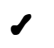 & 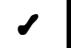 & 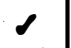 & & & \\
\hline Technical Women's Symposium & & $\mathcal{L}$ & & & & & & & & 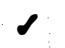 & & & & & & & & & & & & & & . \\
\hline \multicolumn{25}{|l|}{ DOE Lab Roviow of Programs for Women } \\
\hline $\begin{array}{l}\text { \# participants in 2nd Review } \\
\text { Funds from Programs/Overhead } \\
\text { (P/O) }\end{array}$ & 2 & 5 & $\begin{array}{l}5 \\
0\end{array}$ & $\begin{array}{l}1 \\
0\end{array}$ & 0 & $\begin{array}{l}5 \\
0\end{array}$ & $\begin{array}{l}2 \\
0\end{array}$ & $\begin{array}{l}5 \\
0\end{array}$ & $\begin{array}{c}6 \\
\text { PO }\end{array}$ & $\begin{array}{c}7 \\
H F\end{array}$ & $\begin{array}{l}1 \\
0\end{array}$ & & $\begin{array}{c}2 \\
\mathrm{PO}\end{array}$ & $\begin{array}{l}2 \\
P\end{array}$ & $\begin{array}{l}6 \\
P\end{array}$ & 3 & $\begin{array}{l}3 \\
0\end{array}$ & $\begin{array}{c}1 \\
\mathrm{PO}\end{array}$ & $\begin{array}{l}3 \\
0\end{array}$ & $\begin{array}{c}5 \\
\text { HF }\end{array}$ & $\begin{array}{l}6 \\
0\end{array}$ & & $\begin{array}{l}6 \\
0\end{array}$ & 1 \\
\hline $\begin{array}{l}\text { * participants in 3rd Review } \\
\text { Funds from Programs/Overhead } \\
\text { (P/O) }\end{array}$ & 5 & 5 & $\begin{array}{l}4 \\
0\end{array}$ & $\begin{array}{l}2 \\
0\end{array}$ & 1 & $\begin{array}{l}3 \\
0\end{array}$ & 2 & $\begin{array}{c}4 \\
P O\end{array}$ & $\begin{array}{c}4 \\
P O\end{array}$ & 5 & 3 & $\begin{array}{c}7 \\
\text { HF }\end{array}$ & $\begin{array}{c}2 \\
\mathrm{PO}\end{array}$ & $\begin{array}{l}12 \\
\mathrm{HF}\end{array}$ & $\begin{array}{l}19 \\
\text { HF }\end{array}$ & 4 & $\begin{array}{l}4 \\
0\end{array}$ & $\begin{array}{c}4 \\
\text { PO }\end{array}$ & $\begin{array}{l}2 \\
0\end{array}$ & $\begin{array}{l}5 \\
0\end{array}$ & $\begin{array}{l}5 \\
0\end{array}$ & 1 & $\begin{array}{l}1 \\
0\end{array}$ & 1 \\
\hline \# participants expected in 4th Review & 0 & 7 & 5 & 3 & 4 & 1 & 1 & HF & 6 & 7 & 1 & 5 & 7 & 5 & 9 & 3 & 7 & 2.4 & 2 & 6 & HF & 1 & 1 & \\
\hline $\begin{array}{l}\text { Women in Science/Technology booklet: } \\
\text { year issued }\end{array}$ & & 94 & 92 & & & & & & & 92 & & & & & $\begin{array}{l}1 \\
93\end{array}$ & & & & & 94 & 24 & & & \\
\hline $\begin{array}{l}\text { Women in Science/Technology video: } \\
\text { year produced }\end{array}$ & & & Pn & & & & & s. & & & & & & & & & & & & 82 & & & & \\
\hline
\end{tabular}




\section{Appendix D}

\section{Educational Programs}


Educatlon Programs

Pre-College

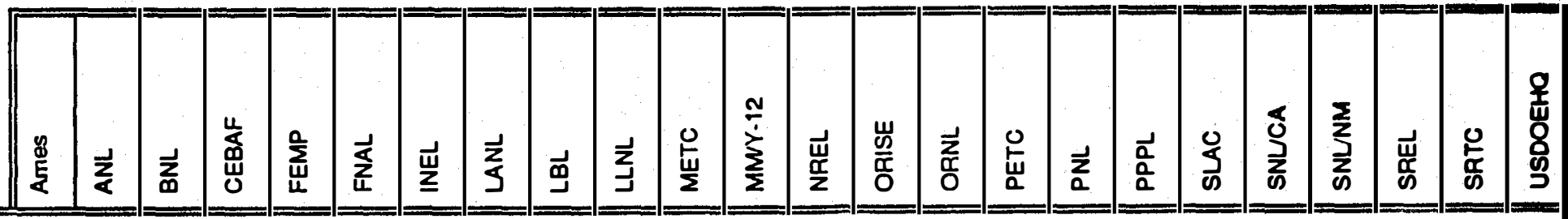

\section{Pro-Collogo Programs - Targoting Young Women}

\begin{tabular}{|c|c|c|c|c|c|c|c|c|c|c|c|c|c|c|c|c|c|c|c|c|c|c|c|}
\hline Take Our Daughters to Work Day & & $\infty$ & 1 & 1 & 1 & & 1 & 1 & 1 & 1 & & 1 & 1 & 1 & cd & 1 & 1 & 1 & 1 & 1 & 1 & & 1 \\
\hline Research Participation & & & $\boldsymbol{J}$ & & & & & & & & & & & & & & & & & & & & \\
\hline Expanding Your Horizons & $\mathbf{p}$ & & & $p$ & us & 1 & & 1 & & $p$ & 1 & & 1 & & & & $\mathbf{p}$ & & 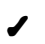 & 1 & J & & \\
\hline Career Days & & & 1 & $p$ & & & & e & & & & & us & 1 & 1 & & & $\alpha$ & $\mathbf{p}$ & & & $\mathbf{p}$ & \\
\hline Young Women's Conference & & J & & & & & 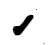 & & & & & & & 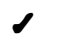 & e & & & & & & & & \\
\hline Summer Camps & & & & $\mathbf{p}$ & & & & & & & & & us & \& & e & & $\mathbf{p}$ & & & & & & \\
\hline Work Study/ntemships & & & 1 & & 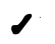 & l & & & & & & & & & & & & & & & & & \\
\hline PreFreshman Enrichment Program & & & & & & & & & & & & & & & & & & & & & & & 0 \\
\hline Summer Institules & & $e$ & & $\mathbf{p}$ & & & & & & & & & & & 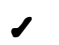 & & & us & & & & & \\
\hline
\end{tabular}

\section{Pro-Colloge Programs - Non Targoted}

\begin{tabular}{|c|c|c|c|c|c|c|c|c|c|c|c|c|c|c|c|c|c|c|c|c|c|c|c|c|}
\hline Research Participation & 8 & 1 & 1 & $\alpha$ & 8 & 1 & 1 & 1 & 1 & 1 & 1 & 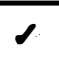 & 1 & 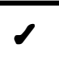 & 1 & 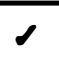 & 1 & 1 & 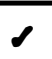 & $\alpha$ & 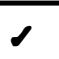 & 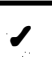 & 8 & \\
\hline Workshops and Institutes & 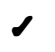 & 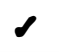 & 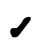 & $\alpha$ & 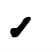 & $\alpha$ & 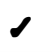 & 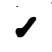 & 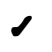 & 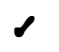 & & & 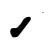 & 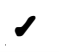 & 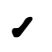 & 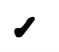 & 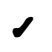 & $\alpha$ & 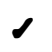 & $\alpha$ & 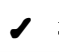 & $\checkmark$ & 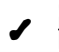 & \\
\hline Instructional Materials & 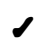 & $\alpha$ & 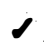 & $\alpha$ & 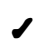 & $\alpha$ & 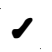 & 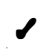 & 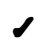 & $\alpha$ & 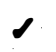 & & & $\alpha$ & 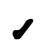 & $\alpha$ & 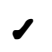 & 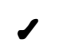 & $\alpha$ & 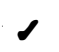 & 1 & 1 & 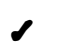 & \\
\hline In-House Tours & 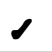 & $\alpha$ & 1 & 1 & 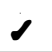 & $\alpha$ & 1 & 1 & 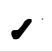 & 1 & & & & 1 & 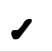 & $\alpha$ & 1 & 1 & 1 & 1 & 1 & 1 & 1 & \\
\hline Direct Classroom Instruction & 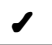 & $\alpha$ & 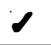 & 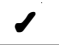 & 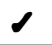 & 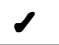 & 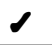 & 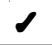 & 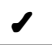 & 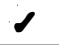 & 1 & & 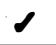 & 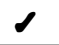 & $\alpha$ & 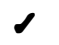 & $\alpha$ & $\alpha$ & $\alpha$ & 1 & / & ${ }^{\prime \prime}$ & $\mathbf{p}$ & \\
\hline Community Outreach & 1 & 1 & 1 & 1 & & 1 & 1 & 1 & $\alpha$ & 1 & 1 & & 1 & 1 & 1 & 1 & 1 & 1 & 1 & 1 & 1 & 1 & 1 & 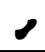 \\
\hline Special Events & 1 & $\alpha$ & $\alpha$ & $\alpha$ & 1 & 1 & 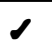 & 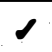 & $\alpha$ & $d$ & 1 & & 1 & $\alpha$ & 1 & 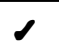 & 1 & 1 & 1 & 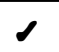 & 1 & 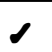 & 1 & 1 \\
\hline
\end{tabular}

co for all children and dependents 
Education Programs College

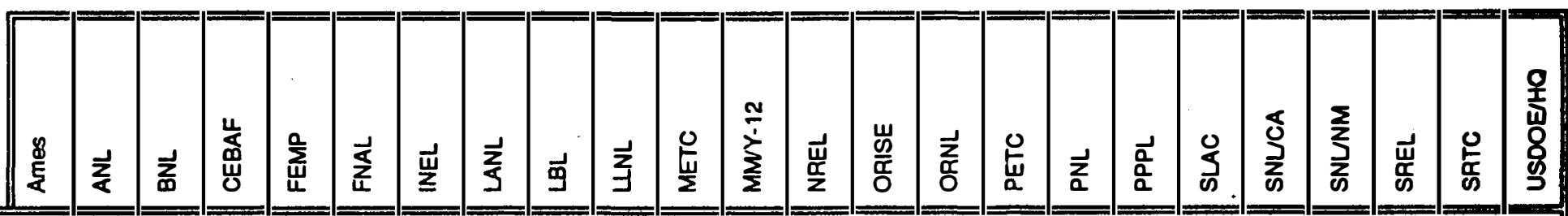

\section{College Programs - Targoting Women}

Research Participation

Summer Science Intern

Graduate Mentoring

Women in Science Conference

College Programs - Non Targeted

Graduate Research Participation

Graduate Research Participation

Undergraduate Research Participation

Faculty Research Participation

Targeted Fellowships

Scientist/Faculty Exchange

Graduate School Fair

- targeted for women and minorities

P participate in programs at local universities \& other organizations 


\section{Appendix E}

\section{List of Participating Facilities}




\section{Llst of Facilitles}

$\begin{array}{ll}\text { Ames } & \text { Ames Laboratory, lowa State University, Ames, IA } \\ \text { ANL } & \text { Argonne National Laboratory, Argonne, IL } \\ \text { BNL } & \text { Brookhaven National Laboratory, Upton, NY } \\ \text { CEBAF } & \text { Continuos Electron Beam Accelerator Facility, Newport News, VA } \\ \text { FEMP } & \text { Fernald Environmental Management Project, Cincinnati, OH } \\ \text { FNAL } & \text { Fermi National Accelerator Laboratory, Batavia, IL } \\ \text { INEL } & \text { EG\&G, Idaho Nuclear Engineering Laboratory, Idaho Falls, ID } \\ \text { LANL } & \text { Los Alamos National Laboratory, Los Alamos, NM } \\ \text { LBL } & \text { Lawrence Berkeley Laboratory, Berkeley, CA } \\ \text { LLNL } & \text { Lawrence Livermore National Laboratory, Livermore, CA } \\ \text { METC } & \text { Morgantown Energy Technology Center, Morgantown, WV } \\ \text { MMN-12 } & \text { Martin Marietta, Y-12 Plant, Oak Ridge, TN } \\ \text { NREL } & \text { National Renewable Energy Laboratory, Golden, CO } \\ \text { ORISE } & \text { Oak Ridge Institute for Science and Education, Oak Ridge, TN } \\ \text { ORNL } & \text { Oak Ridge National Laboratory, Oak Ridge, TN } \\ \text { PNL } & \text { Pacific Northwest Laboratory, Richland, WA } \\ \text { PETC } & \text { Pittsburgh Energy Technology Center, Pitsburgh, PA } \\ \text { PPPL } & \text { Princeton Plasma Physics Laboratory, Priceton, NJ } \\ \text { SLAC } & \text { Stanford Linear Accelerator Center, Stanford, CA } \\ \text { SNLCA } & \text { Sandia National Laboratories, Org. 8284, Livermore, CA } \\ \text { SNLNM } & \text { Sandia National Laboratories, Org. 6600, Alburquerque, NM } \\ \text { SREL } & \text { Savannah River Ecology Laboratory, Aiken, SC } \\ \text { SRTC } & \text { Savannah River Technology Center, Aiken, SC } \\ \text { USDOE/HQ } & \text { U.S. Dept. of Energy, Washington, DC }\end{array}$

